\title{
ASPECTOS DA ATIVIDADE ASSISTENCIAL DA ENFERMEIRA NA UNIDADE DE INTERNAÇÃO PEDIÃTRICA *
}

\author{
Autora: Dulce Maria Nunes ** \\ Co-Autoras: Helena Becker Issi *** \\ Nair Regina R. Ribeiro **** \\ Maria da Graça C. da Motta **
}

ReBEn/05

NUNES, D.M. e Colaboradoras - Aspectos da Atividade Assitencial da Enfermeira na Unidade de Internação Pediátrica. Rev. Bras. Enf.: RS, 36: 29-37 , 1983.

Neste trabalho, entende-se que:

ATIVIDADE - é o conjunto de operações e movimentos independentes que objetivam atingir um fim determinado ${ }^{1}$.

DESEMPENHO - é a execução, o exercício, a prática de uma função ${ }^{1}$.

FUNÇÃO -- é a atribuição, ou conjunto de atribuições, conferido a cada categoria profissional ou cometida individualmente, a deternninados servidores e ou empregados, para execução de determinadas atividades 1 .

\section{RESUMO}

O trabalho faz uma abordagem geral sobre as atividades assistenciais da enfermeira na Unidade de Internação Pediátrica.

Tem como objetivo propor, aos enfermeiros que desempenham suas funções em Enfermagem Pediátrica Hospitalar, um diálogo no sentido de conhecer como essas atividades são desenvolvidas nas diversas regiōes do País e discutir experiências.

Como ponto de referência, relatam-se as atividades desenvolvidas na Unidade de Internação Pediátrica do Hospital de Clínicas de Porto Alegre, RS, considerando os objetivos da Unidade Pediátrica, o Sistema de Permanência Conjunta e a Equipe Interdisciplinar.

Conclui-se que, desenvolvendo tais atividades, a enfermeira assegurará ao paciente pediátrico hospitalizado, e a sua fam llia, assistência contínua e integral.

" Trabalho apresentado no XXXIV Congresso Brasileiro de Enfermagem. Porto Alegre, RS/ 24 a 29 de outubro de 1982.

: * Professora Assistente do Departamento de Enfermagem Materno-Infantil, da Escola de Enfermagem da Universidade Federal do Rio Grande do Sul.

***: Professora Horista do Departamento de Assistência e Orientação Profissional da Escola de Enfermagem da Universidade Federal do Rio Grande do Sul. Enfermeira Pediatra, Chefe da Unidade de Internação Pediátrica Sul, do Hospital de Clínicas de Porto Alegre, RS.

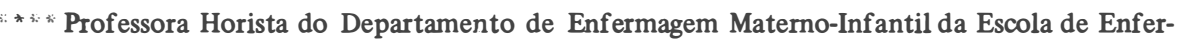
magem da Universidade Federal do Rio Grande do Sul. Enfermeira Pediatra, Chefe da Unidade de Tratamento Intensivo Pediátrico, do Hospital de Clínicas de Porto Alegre, RS. 
NUNES, D.M. e Colaboradoras - Aspectos da Atividade Assitencial da Enfermeira na Unidade de Internação Pediátrica. Rev. Bras. Enf.: RS, 36: 29-37 , 1983.

Recomenda-se esforço, por parte dos enfermeiros, para que sejam estabelecidos os objetivos das atividades de enfermagem nas Unidades de Internação Pediátricas, critérios definidos e, em especial, operacionalização inteligente e criativa do tempo no desempenho de suas funções.

\section{INTRODUÇÃO}

A razão deste trabalho é discutir os elementos que poderão fazer parte de um plano de cuidados à criança hospitalizada, enfatizando os aspectos mais relevantes da atividade da enfermeira na Unidade de Internação Pediátrica.

Uma abordagem determinante da assistência de enfermagem pediátrica é a consciência da enfermeira ao agir em função das necessidades peculiares da criança e sua família. A respeito disto, HENDERSON ${ }^{3}$ crê que "a função que nós acreditamos desempenhada pela enfermeira é primariamente uma função independente - a de agir pelo paciente onde este não tenha conhecimento, força física ou o desejo de agir em seu próprio favor. Encaramos esta função como complexa e criativa, oferecendo oportunidade ilimitada para a aplicação das ciências físicas, biológicas e sociais, bem como o desenvolvimento de habilidades que sobre ela se baseiam".

A onganização das atividades assistenciais e educativas na Unidade de Internação Pediátrica depende da filosofia da instituição em relação à criança doente, da definição de critérios para a assistência de enfermagem, da existência de funções definidas para cada profissional de uma equipe interdisciplinar, do número de pacientes em relação ao número de profissionais e categorias ocupacionais, e do objetivo da instituição.

Detendo-se nesses fatores, é importante que a enfermeira considere suas funções e, dentro destas, suas atividades, assumindo a determinação da quantidade e qualidade das mesmas, no contexto de seu desempenho.

Essas atividades, selecionadas e analisadas criteriosamente, possibilitam à enfermeira assegurar ao paciente pediátrico hospitalizado, e sua família, assistência contínua e integral.

Sabe-se que existem metodologias já convencionais para a elaboração de planos assistenciais, porém os passos que as compõem, na maioria das vezes, não são desenvolvidos devido ao número e qualidade dos recursos humanos de que dispõem as instituições hospitalares.

Como refere CIUCA2 : "Um plano de enfermagem é um plano personalizado, corretamente escrito para um paciente em particular, indicando o tipo de enfermagem que ele necessita, como pode ser melhor realizado e as metas que o pessoal de enfermagem espera alcançar com o paciente".

É preciso que se conheça a criança, para que se possa agir em enfermagem pediátrica, sendo função da enfermeira, através do uso de seus instrumentos básicos, o estabelecimento da assistência às necessidades da criança.

Em se tratando de prestar assistência, PAIM $^{5}$ coloca com propriedade a extensão da mesma que a enfermagem deve atingir: "a tendência de assistir o paciente, a partir da identificação de problemas e deteç̧ão de necessidades em níveis psicobiológicos, psicossociais e psicoespirituais, vem substituindo, na atualidade, aquele enfoque que se dava à doença. A literatura demonstra a busca, pelos estudiosos, da sistematização dos conhecimentos científicos e das próprias ações de enfermagem".

Entende-se que, se os enfermeiros que desempenham suas funções em Enfermagem Pediátrica Hospitalar estabelecessem um plano assistencial mínimo, adaptado às condições individuais de cada criança, possibilitariam assistência integral ao paciente pediátrico, levando em conta que para isto não se pode dissociar o binômio criança/fam ília. 
NUNES, D.M. e Colaboradoras - Aspectos da Atividade Assitencial da Enfermeira na Unidade de Internação Pediátrica. Rev. Bras. Enf.: RS, 36: 29-37 , 1983.

\section{ATIVIDADE ASSISTENCIAL DA ENFERMEIRA NA UIP DO HCPA - RS}

$\mathrm{Na}$ tentativa de partir para um tipo de assistência mais completa, de modo que a enfemeira na Unidade de Internação Pediátrica do Hospital de Clínicas de Porto Alegre, RS, tivesse suas atividades centradas nas necessidades da criança doente e de sua família, estabeleceu-se uma metodologia para o desenvolvimento das atividades da enfermeira, em um turno ou mais.

A Unidade de Internação tem como objetivo prestar assistência integral à criança, sem dissociá-la do contexto familiar, através da promoção de meios para a recuperação da saúde, capacitação da família para a manutenção desta e orientação para o autocuidado.

A Unidade de Pediatria tem uma capacidade de 80 leitos clínicos e cirúrgicos, para crianças de 60 dias a 12 anos.

A área física é dividida em Unidade de Emergência, porta de entrada para todo paciente que se interna na Pediatria; Unidade de Terapia Intensiva, Unidade de Internação Norte, e Unidade de Internação Sul.

A Unidade de Pediatria possui um sistema de Permanência Conjunta, em função do qual a equipe interdisciplinar pediátrica fundamentou a atitude de interação afetiva e educação para a saúde, visando proporcionar à criança/famllia uma assistência integral.

A equipe interdisciplinar que assiste a criança e sua famılia, diariamente, segundo as categorias, consta de:

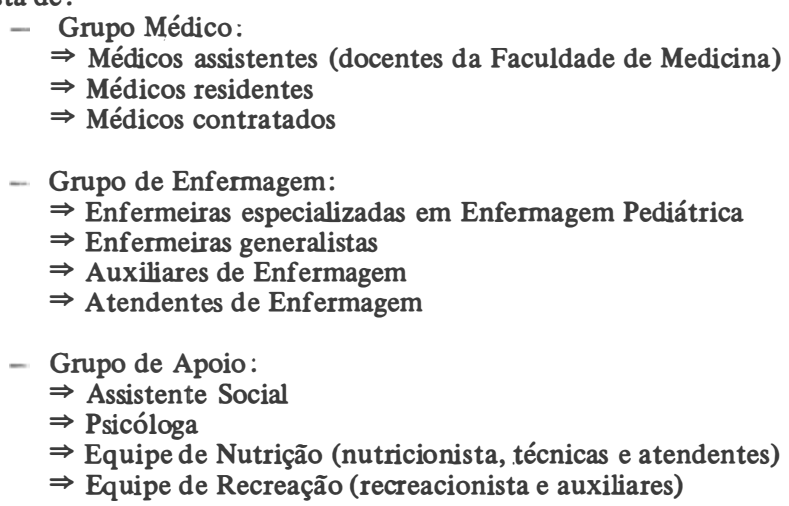

- Os Pais: Considera-se os pais elementos integrantes da equipe, visto que os mesmos parcipam no cuidado de seus filhos.

Dentro desta equipe, a enfermeira tem a função de prestar assistência de enfermagem, através da identificação, interpretação e avaliação das necessidades da criança hospitalizada e sua famûlia, planejar, executar, registrar, analisar e reavaliar as ações de enfermagem prestadas; colaborar com os outros elementos da equipe interdisciplinar, através de sua observação constante, e, principalmente, educar para a saúde.

Segundo PAIM, HOELZ \& CASTRO4:

"O enfermeiro lidera sua equipe, é responsável pelas ordens de enfermagem do plano de cuidados do paciente, quer no aspecto de indicação da ordem como prerrogativa exclusiva, quer no aspecto de supervisão e execução de cuidados de enfermagem. Isto implica a mais profunda responsabilidade quanto à assistência prestada ao paciente, visto que essa assistência provém do registro das intervenções, por ele indicadas na qualidade de ordens de enfermagem ou prescrições de enfermagem, unidades componentes dos planos de cuidados".

seguintes passos:

Para proceder às etapas que compõem a assistência de enfermagem, são desenvolvidos os 
NUNES, D.M. e Colaboradoras - Aspectos da Atividade Assitencial da Enfermeira na Unidade de Internação Pediátrica. Rev. Bras. Enf.: RS, 36: 29-37 , 1983.

\section{PASSAGEM DE PLANTÃO}

A passagem de plantão é uma forma de comunicação em enfermagem, consistindo na tranferiência dos planos de cuidados (asistência) de enfermagem da equipe de um turno para a do outro turno.

Cabe à enfermeira que entrega o turno, a liderança desse trabalho. Ela reúne as duas equipes, com todas as informações de cada paciente organizadas e registradas, todos os elementos da equipe que assistiram os pacientes deverão participar ativamente com o relato verbal, sucinto, das ocorrências com seu paciente.

À enfermeira que recebe o plantão cabe:

1.1 - Avaliação das condições gerais dos pacientes

1.1.1 - Condições vitais

1.1.2 - Necessidades básicas

1.1.3 - Unidade do paciente

A identificação e interpretação das situações acima possibilitam à enfermeira avaliar seus recursos humanos e técnicos e, através deles, planejar e operacionalizar as atividades assistenciais e educativas do seu turno.

1.2 - Distribuição das tarefas delegáveis à sua equipe, considerando as prioridades da criança/fam llia, tais como risco, condições de preferência da criança/funcionário, habilidades e segurança do funcionário.

1.3 - Orientação individual ou coletiva da equipe, esclarecendo cada elemento como deverá desenvolver as tarefas, enfatizando os cuidados específicos.

1.4 - Observações especiais - consideram-se como tais aquelas que envolvem a atenção de toda a equipe de enfermagem, pois que, em determinado momento, poderá ser necessária a intervenção de qualquer elemento da equipe. Ex.: paciente com distúrbio de conduta.

\section{AVALIAÇÃO SUMÅRIA DAS CONDIÇÕES DA CRIANÇA (EXAME FÍSICO)}

Conhecendo as necessidades individuais de cada criança, a enfermeira planejará ou reformulará a conduta de enfermagem.

2.1 - Com a realização sumária do exame físico, a enfermeira pode orientar-se sobre o estado geral da criança, detectando e prevenindo problemas, visando a assistência individualizada.

2.2 - A utilização dos registros do turno antecedente é outro elemento fidedigno para a orientação do plano continuado. O registro objetivo e conciso of erece informações indispensáveis (o que se verá no item correspondente).

2.3 - Informações da criança/famllia - as informações verbalizadas pela criança e pela família constituem também elementos importantíssimos na elaboração dos planos de cuidado, possibilitando à enfermeira detalhes mais subjetivos.

\section{REAVALIAÇÃO DOS PLANOS DE CUIDADO E PROPOSIÇÃO DE NOVAS CONDUTAS}

Significa elaborar uma nova prescrição, revisar ou manter a anterior. A condição atual da criança é que determinará o plano de cuidados.

Na elaboração deste, poderão ser seguidos os seguintes critérios:

3.1 - Problema principal da criança - consiste em controlar as alterações fisiopatológicas oriundas da patologia específica.

Problemas secundários - trata-se de controlar as alterações secundárias que possam advir do problema principal ou outros.

Condições vitais - acompanhar e avaliar, através de controle, de acordo com as necessidades do paciente.

3.2 - Controle de peso e medidas - caso se aplique

3.3 - Controle das condições psicoafetivas da criança e famıllia

3.4 - Execução da terapêutica médica

3.5 - Controle específico da terapêutica - efeitos e para-efeitos

3.6 - Rotinas de controle de ingesta e eliminações

3.7 - Rotinas de higiene

3.8 - Atividades recreativas

3.9 - Encaminhamento a outros profissionais da equipe interdisciplinar 
NUNES, D.M. e Colaboradoras - Aspectos da Atividade Assitencial da Enfermeira na Unidade de Internação Pediátrica. Rev. Bras. Enf.: RS, 36: 29-37 , 1983.

Observe-se que os cuidados devem ser prescritos em ordem de prioridade, de acordo com os problemas que requerem assistência imediata.

O desenvolvimento da prescrição é avaliada pela enfermeira junto com o funcionário, no momento da orientação.

$\mathrm{O}$ aprazamento dos controles e rotinas também é determinado pela enfermeira, bem como a alteração dos mesmos.

\section{TÉCNICAS NÃO-DELEGÃVEIS}

Constituem-se de procedimentos que utilizam recursos básicos de enfermagem, tais como: aplicação de princípio científico, destreza manual, criatividade e bom senso, razão pela qual não devem ser delegados.

São técnicas mais elaboradas, não-rotineiras, e cuja execução, muitas vezes, pode ser determinada por uma necessidade urgente da criança. São atividades que a enfermeira, sempre que possível, não deve delegar. Exigem dela o acompanhamento criterioso da situação, observando a reação física e emocional da criança. riscos e recursos.

Outras implicações relacionadas com o desenvolvimento destas técnicas são: local, idade,

\section{SUPERVISÃO}

5.1 - Direta - Faz-se pelo acompanhamento da evolução das condições da criança pela enfermeira, observando reações e desenvolvendo procedimentos e controles juntamente com os demais elementos de sua equipe.

5.2 - Indireta - Faz-se pelo acompanhamento da evolução das condições da criança, através das observações e controles executados somente pela sua equipe.

\section{PROGRAMA EDUCATIVO}

6.1 - Orientação quando da admissão da criança/fam 11ia

É importante que a famllia receba e sinta, desde o início, uma atenção individualizada, e a orientação na admissão é um dos primeiros passos para que tal suceda. É fundamental que os pais percebam quão importantes são para o tratamento e recuperação de seus filhos; por isso devem ser chamados a integrar, como elementos participantes, a equipe de saúde.

Note-se, porém, que é preciso orientar os pais para a forma certa de agir, considerando-se as condições da criança, o nível de ansiedade a que estão submetidos pela doença do fillho, assim como suas condições sócio-econômicas e culturais. Para que não se sintam perdidos, sua colaboração deverá ser solicitada segundo critérios previamente estabelecidos:

$20 \mathrm{~h})$;

- dàndo-lhes conhecimento do horário em que podem permanecer junto ao fillho (das 8 às solicitados, como na:

- indicando-lhes o tipo de auxílio que podem prestar no tratamento da criança, quando

$\Rightarrow$ alimentação: ressalta-se a importância de a criança somente receber os alimentos preparados pelo Serviço de Nutrição do hospital, excetuando-se os casos em que a recusa alimentar contínua possa interferir no pronto restabelecimento. Sugere-se que, sempre que a mãe estiver presente, respeitadas as condições da mesma e as da criança, aquela administre a alimentação ao fillho, segurando-o ao colo e aproveitando o momento para estreitar a ligação af etiva entre ambos.

$\Rightarrow$ higiene: a mãe, quando presente, é estimulada a dar o banho e a trocar as roupinhas e fraldas da criança. Aproveita-se o momento para inserir ou dar continuidade aos hábitos rotineiros, lembrando-a para trazer a escova de dentes e o pente da criança.

$\Rightarrow$ hábitos: solicita-se à mãe que informe os principais hábitos da criança, para sua melhor adaptação ao hospital, tais como o uso de dois bicos, de cheirar paninhos, ou de fralda para dormir; o gosto de ouvir cantigas ou estórias; a reza ao dormir; o nome/apelido pelo qual prefere ser chamado, e outros.

$\Rightarrow$ recreação: a mãe é convidada para que aproveite integralmente o tempo de permanência junto ao filho, para servir-lhe de estímulo e apoio. Sugerimos que converse e brinque com ele enquanto estiver no leito, quarto, enfermaria ou na sala de recreação. A mãe, orientada, pode auxiliar as recreacionistas a of erecer os brinquedos à criança, e pode trazer de casa aquele que for o seu preferido. 
NUNES, D.M. e Colaboradoras - Aspectos da Atividade Assitencial da Enfermeira na Unidade de Internação Pediátrica. Rev. Bras. Enf.: RS, 36: 29-37 , 1983.

$\Rightarrow$ visitas: solicita-se a colaboração dos pais para que, durante o período de visitas, permaneçam somente duas pessoas de cada vez junto à criança, e que estas pessoas estejam com boa saúde (pretende-se com isto manter um ambiente tranqüilo e seguro para a criança, do ponto de vista emocional e físico).

$\Rightarrow$ proteção de acidentes: os pais tranquilizam-se ao saber que a equipe está atenta à proteção e segurança da criança, e eles próprios são alertados para alguns pontos de risco, que, com sua observação, auxiliam a prevenir, como: as grades da cama deverão ser elevadas quando se afastarem da criança; as portas dos quartos e corredores laterais devem permanecer fechadas; objetos pontiagudos ou cortantes devem ser mantidos fora do alcance das crianças, o funcionário responsável deve ser avisado quando os pais precisam se afastar, evitando que a criança fique sozinha no quarto ou enfermaria.

- dando-lhes conhecimento das rotinas da Unidade, para que colaborem com sua dinâmica de funcionamento, por exemplo:

$\Rightarrow$ visitas de crianças menores de 10 anos devem ser autorizadas pelas enfermeira da Unidade;

$\Rightarrow$ refeições ou lanches podem ser obtidos no refeitório ou no bar do hospital, evitando assim de se alimentarem nas próprias enfermarias ou quartos;

$\Rightarrow$ locais especiais podem ser utilizados para fumar ou para um bate-papo com outros pais,

$\Rightarrow$ o telefone do Posto de Enfermagem é de uso interno da Unidade, havendo um telefone público na Unidade que pode ser utilizado.

- convidando-os a participarem dos programas de educação para a saúde.

\section{2 - Entrevistas individuais}

O histórico do desenvolvimento é realizado através de entrevista da enfermeira com a mãe da criança.

Parte-se sempre da prioridade da familia/criança (itens do Histórico do Desenvolvimento: Identificação, Período Pré-Natal; Período Pós-Natal; Alimentação e Hidratação; Eliminações; Sono e Repouso; Desenvolvimento Neuro-Psicomotor; Hábitos de Saúde e Higiene; Antecedentes Mórbidos; Antecedentes Médico Familiares; Percepções e Expectativas).

Ao concluir a entrevista, selecionam-se os problemas que fazem parte do grupo de prioridades. Os problemas que exigem terapêutica de enfermagem ao longo da internação são aprazados para entrevistas subseqüentes e os que não são de enfermagem são encaminhados ao respectivo profissional da equipe.

\section{3 - Palestras}

As palestras são realizadas pelas enfermeiras, para grupos de pais e outros familiares que queiram participar.

Do programa, fazem parte os temas solicitados pelos pais, assim como aqueles em que fique evidenciada - através do comportamento paterno/materno - a conveniência da abordagem. $O$ fornecimento de orientação para prevenir determinadas patologias mais comuns pode ditar alguns temas do programa e, sobretudo, a necessidade de educar para a saúde.

São realizadas, quando possível, diariamente, alternando os turnos (manhã-tarde).

A participação dos pais é observada e registrada, incluindo-se o aproveitamento segundo o feedback realiz ${ }^{2}$ o pela palestrante.

As palestras coletivas também fazem parte de terapêutica de enfermagem.

Conteúdo.

O plano de palestras inclui as seguintes etapas: Objetivos, Técnica de ensino, Recursos e

\section{4 - Reforços educativos para a alta hospitalar}

Em continuidade à terapêutica de enfermagem hospitalar, são incluídos aspectos educativos de reforços a serem realizados no momento anterior à saída da criança do hospital.

Esse procedimento de orientação para a alta hospitalar tem por fim dar continuidade ao tratamento da patologia; manter controle periódico de saúde; prevenir recidivas, educar para a saúde abrangendo os aspectos biopsicossociais da criança; reforçar as orientações realizadas durante a internação e também os encaminhamentos. 
NUNES, D.M. e Colaboradoras - Aspectos da Atividade Assitencial da Enfermeira na Unidade de Internação Pediátrica. Rev. Bras. Enf.: RS, 36: 29-37 , 1983.

\section{Como proceder:}

- A enfermeira investiga, junto ao familiar, seu conhecimento a respeito da patologia e os aspectos de manutenção da saúde do filho. Partindo deste conhecimento, inicia as orientações sobre:

$\Rightarrow$ o que é a doença e aspectos preventivos da mesma

$\Rightarrow$ cuidados

$\Rightarrow$ tratamento

$\Rightarrow$ atividades específicas

$\Rightarrow$ retornos

Ela deverá ainda, seguindo um roteiro preestabelecido, reforçar aspectos ligados à educa ção para a saúde, com os seguintes tópicos:

- Controle de Saúde $\left\{\begin{array}{l}\text { Consultas periódicas } \\ \text { Imunizações }\end{array}\right.$

$\begin{aligned} & \begin{array}{l}\text { - Alimentação } \\ \text { Nutricionista) }\end{array}\left\{\begin{array}{l}\text { Higiene } \\ \text { Técnica } \\ \text { Seleção dos alimentos } \\ \text { Estimulação para a auto-alimentação }\end{array}\right. \\ & \text { - Higiene }\left\{\begin{array}{l}\text { Corporal } \\ \text { Vestuário } \\ \text { Estimulação do autocuidado }\end{array}\right. \\ & \text { - Recreação }\left\{\begin{array}{l}\text { Atividade ao ar livre } \\ \text { Banho de sol } \\ \text { Brinquedos adequados para a idade }\end{array}\right. \\ &\left\{\begin{array}{l}\text { Psicomotora }\left\{\begin{array}{l}\text { Posicionamento } \\ \text { Visual } \\ \text { Sonora } \\ \text { Tátil }\end{array}\right. \\ \text { Afetiva }\end{array}\right.\end{aligned}$

- Sono e Repouso $\left\{\begin{array}{l}\text { Período conforme idade } \\ \text { Ambiente }\end{array}\right.$

- Condições de Moradia $\left\{\begin{array}{l}\text { Higiene } \\ \text { Ventilação }\end{array}\right.$

- Ambiente Familiar $\left\{\begin{array}{l}\text { Relacionamento dos pais entre si } \\ \text { Relacionamento dos pais com a criança } \\ \text { Relacionamento dos pais com outros membros da famllia }\end{array}\right.$

Por fim, cabe ainda à enfermeira solicitar as impressões do familiar com referência ao período de internação, tais como:

- Aspectos negativos

- Aspectos positivos

- Encaminhamentos 
NUNES, D.M. e Colaboradoras - Aspectos da Atividade Assitencial da Enfernneira na Unidade de Internação Pediátrica. Rev. Bras. Enf.: RS, 36: 29-37 , 1983.

Toda a orientação da alta hospitalar deverá constar nos registros:

$\Rightarrow \mathrm{Na}$ folha de evolução, os itens orientados

$\Rightarrow$ As informações fornecidas pelos familiares

\section{REGISTROS}

O Hospital de Clínicas de Porto Alegre tem um sistema de prontuário único, ou seja, todos os profissionais usam a mesma metodologia de registro: sistema de Weed, de registro orientado para o problema.

$\AA$ enfermeira, em cada turno, cabe o registro de toda e qualquer assistência prestada ao paciente/família. Um dos aspectos para o qual se chama a atenção é a evolução de enfermagem, seguindo os aspectos:

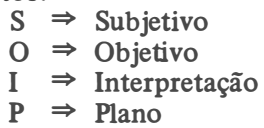

- Subjetivo

$\Rightarrow$ São as informações fornecidas pela criança ou familiares importantes para o tratamento e orientação da equipe;

$\Rightarrow$ os sentimentos, queixas, necessidades biopsicossociais manifestadas pela criança.

- Objetivo

Aqui se incluem as observações feitas através dos sentidos do profissional:

$\begin{array}{ll} & \text { estado vital } \\ & \text { estado nutricional } \\ & \text { hidratação } \\ & \text { ingestas e excretas } \\ & \text { oxigenação } \\ \text { condições circulatórias } \\ \text { alterações e estado } \\ \text { condiçes do sensório } \\ \text { condições de pele e mucosas }\end{array}$

$\Rightarrow$ alterações detectadas no exame físico diário

$\Rightarrow$ aspectos do desenvolvimento psicomotor

- Interpretação

É a síntese do Subjetivo e do Objetivo através da avaliação da enfermeira.

- Plano

São os aspectos mais importantes da conduta de enfermagem a serem desenvolvidas com a criança e a famńlia:

$\Rightarrow$ orientações à criança e /ou famulia

$\Rightarrow$ encaminhamentos aos demais profissionais

$\Rightarrow$ procedimentos mais elaborados

$\Rightarrow$ aspectos que servem de reforço à prescrição de enfermagem

Em uma unidade pediátrica onde exista a permanência de pais com a criança, chama-se a atenção para o registro objetivo da observação sistematizada das condições físicas, emocionais e espirituais da criança, e a integração com a famnlia, possibilitando maiores subsídios para uma interpretação global das necessidades do binômio criança/familia.

Como refere ROCHA 6 , "em Pediatria a enfermeira deve observar e registrar não apenas o que diz respeito aos sintomas e sinais que evidenciam a evolução da doença, mas também devem ser 
NUNES, D.M. e Colaboradoras - Aspectos da Atividade Assitencial da Enfermeira na Unidade de Internação Pediátrica. Rev. Bras. Enf.: RS, 36: 29-37 , 1983.

observadas as respostas da criança à doença e ao ambiente hospitalar e, mais ainda, as reações dos pais durante todo o tratamento. Só desta forma, a enfermeira conseguirá ajudar a criança a manter-se em equilibrio e, ao mesmo tempo, contribuir para que o grupo familiar supere a ansiedade".

\section{CONCLUSĀO}

As atividades que a enfermeira realiza na Unidade de Internação Pediátrica, que foram relatadas, caracterizam a ação independente, indispensável e não-delegável da enfermeira.

O paciente pediátrico recebe assistência de enfermagem especializada, ininterrupta e completa. A assistência prestada à famillia é simples, sem sofisticação, o que acarreta nela plena satisfação por receber orientação dos profissionais de saúde e, principalmente, por estar no hospital com a criança, participando nos cuidados a esta prestados.

A enfermeira, desenvolvendo atividades não-rotineiras, tem a possibilidade de criar e sentirse gratificada profissionalmente - porque sente o fruto de seu trabalho -, na assistência global à criança, no auxílio à sua recuperação e na educação da família.

\section{RECOMENDAÇŌES}

- Que as enfermeiras se preparem para que tenham condições de realizar as atividades assistenciais e educativas, na Unidade de Internação Pediátrica, com propriedade;

- que realizem suas atividades específicas, não as delegando, para que possam manter a qualidade da assistência e bem definidas suas funções;

- que as experiências adquiridas sejam analisadas, reforçando os aspectos positivos e comuns no desempenho de suas funções.

\section{SUMMARY} vention Unit.

This work is a general approach to the relief activities of the nurse at The Pediatric Inter-

Its main goal is to propose to those nurses performing in hospital pediatric nursing a dialogue to the purpose of understanding how these activities are conducted in our country exchanging viewpoints about their previous experiences.

As a reference, activities developed at the UIP-HCPA-RS are presented, eeping in mind the objectives of the Pediatric Unit, the rooming-in system and the multidisciplinar team.

The conclusion reached is that trough such activities the nurse will be in a position to offer the admitted pediatric patient, as well as to his family, unceasing and whole assistance.

Efforts, on the nurse's side, are recommended to establish specific objectives to the Pediatric Units, well defined criteria to nursing assistance and, above all, creative and intelligent operacionalization of time spent at their functions.

\section{REFERENCIAS BIBLIOGRÁFICAS}

1 - BURLAMAQUE, Clélia Soares. Estudo do desempenho do enfermeiro de um hospital de ensino em nível de unidade de internação. Porto Alegre, UFRGS/Pós-Graduação em Enfermagem, 1981. p. viii. Dissert. (Mest. Enf.).

2 - CIUCA, Rudy L. Over the years with the nursing care plan. Nursing Outlook, New York, 20(11): 706-10, Nov. 1972.

3 - HENDERSON, Virgínia. The nature of nursing. American journal of Nursing, New York, 64(8): 62-8, Aug. 1964.

4 - PAIM, Lygia. A prescrição de enfermagem : unidade valorativa do plano de cuidado. Rio de Janeiro, UFRJ/Escola de Enfermagem Ana Neri, 1976. p. 19-20. Dissert. (Mest. Enf.)

5 - PAIM, Rosalda da Cruz Nogueira. Problemas de enfermagem e terapia centrada nas necessidades do paciente. Rio de Janeiro, União dos Cursos Cariocas, 1978. p. 39.

6 - ROCHA, D. N. Comportamento terapêutico da enfermeira na hospitalização da criança. Revista Brasileira de Enfermagem, Brasilia, 25(1): 89-100, jan/abr. 1972. 